Appears in: Information and Computation, Vol. 110, No. 2, Academic Press, May, 1994, pp 305-326.

\title{
A Partial Approach to Model Checking*
}

\author{
Patrice Godefroid Pierre Wolper \\ Université de Liège \\ Institut Montefiore, B28 \\ 4000 Liège Sart-Tilman, Belgium \\ Email: \{god,pw\}@montefiore.ulg.ac.be
}

\begin{abstract}
This paper presents a model-checking method for linear-time temporal logic that can avoid most of the state explosion due to the modelling of concurrency by interleaving. The method relies on the concept of Mazurkiewicz's trace as a semantic basis and uses automata-theoretic techniques, including automata that operate on words of ordinality higher than $\omega$.
\end{abstract}

\section{Introduction}

Model checking [CES86, LP85, QS81, VW86] is an effective and simple method for verifying that a concurrent program satisfies a temporal logic formula. It works on finite-state programs and proceeds by viewing the program as a structure for interpreting temporal logic and by evaluating the formula on that structure. It is much simpler than temporal deductive proofs and can be easily and effectively implemented.

It has been intensively studied for linear-time temporal logic [LP85, VW86, Var89], branching-time temporal logic [CES86, EL85b, EL85a, Bro86] and temporal $\mu$-calculi [EL86, Var88, Cle90, SW89]. It has been extended to probabilistic [Var85, PZ86, VW86, CY90] as well as real-time programs and logics [ACD90, AH90, HLP90]. It has been adapted to programs containing arbitrary numbers of identical processes [CGB86, CG87, GS87, WL89, KM89]. Methods for making it applicable to very large systems have been investigated

\footnotetext{
${ }^{*}$ This research was supported by the European Community ESPRIT BRA project SPEC (3096).
} 
[BCM ${ }^{+}$90, CMB90, CVWY90, GS90]. Moreover, the results from its experimental use have been very encouraging [RRSV87, BCD85]. What more can be said about it?

In spite of all its success, almost all work around model checking is based on a very wasteful idea: modelling concurrency by interleaving. Even if one is not inclined to loose sleep about whether interleaving semantics are adequate for concurrency, it remains unarguably silly to investigate the concurrent execution of $n$ events by exploring all $n$ ! interleavings of these events!

In this paper, we develop a simple method for applying model checking without incurring most of the cost of modelling concurrency by interleaving. Our method yields results identical to those of methods based on interleaving semantics, it just avoids most of the associated combinatorial explosion. It is quite orthogonal to model checking based on partial-order logics [PW84, KP86, Pen90]. Indeed, these logics are designed to be semantically more powerful. We are "only" more efficient. The idea that the cost of modelling concurrency by interleaving can be avoided in finite-state verification already appears in [PL90, Val91, Val90, God90]. We build upon this earlier work, specifically that of [God90], and bring to it the full capabilities of model checking.

We study model checking for linear-time temporal logic and adopt the automata-theoretic approach of [VW86, Var88, Wol89]. In this approach, the program is viewed as a collection of communicating automata on infinite words [Büc62]. It can thus include arbitrary fairness conditions. The negation of the formula to be checked is then also converted to an automaton on infinite words and the verification can be done by simply checking that the product of the automata describing the program and the automaton corresponding to the negation of the formula is nonempty. This is traditionally done by computing the product automaton which is where the cost of modelling concurrency by interleaving has to be paid.

In [God90] it is shown that the global behavior of a set of communicating processes can be represented by an automaton which can be much smaller than the usual product automaton. The basic idea is to build an automaton that only accepts one interleaving of each concurrent execution. The method is justified by using partial-order semantics, namely the concept of Mazurkiewicz's trace [Maz86] and the automaton is thus called a trace automaton. A trace automaton can be viewed as an automaton accepting at least one, but usually no more than one, interleaving for each trace (concurrent computation) 
of the concurrent program. Thus, together with the independence relation on transitions, this automaton fully represents the concurrent executions of the program. The practical benefit is that this automaton can be much smaller than the automaton representing all interleavings.

The motivating idea behind the method presented here is that, in the automata-theoretic approach to model checking, the trace automaton could be used in place of the product automaton. Unfortunately, this is not directly the case. However, we are able to obtain such a result by using a new type of automaton.

We consider automata operating on infinite words of ordinality higher than $\omega$. Precisely, we define automata operating on words of length $\omega \times n, n \in \omega^{1}{ }^{1}$ We study these automata and show that their emptiness can be efficiently decided. We then show how, when it is viewed as an $\omega \times n$-automaton, the trace automaton can be used to improve the efficiency of model checking.

Finally, we conclude the paper with a comparison between our contributions and related work.

\section{Automata and Model Checking}

We briefly recall the essential elements of the automata-theoretic approach to model checking. More details can be found in [VW86, Wol89, ACW90] and in Chapter 4 of [Tha89]. The problem we consider is the following. We are given a concurrent program $P$ composed of $n$ processes $P_{i}$, each described by a finite automaton $A_{i}$ on countably infinite words over an alphabet $\Sigma_{i}$. We are also given a linear-time propositional temporal logic formula $f$. The model-checking problem is then to verify that all infinite behaviors of the program $P$ satisfy the temporal formula $f$.

The automata we use for describing the processes $P_{i}$ are generalized Büchi automata $^{2}$, i.e. tuples $A=\left(\Sigma, S, \Delta, s_{0}, \mathcal{F}\right)$, where

- $\Sigma$ is a finite alphabet,

- $S$ is a finite set of states,

\footnotetext{
${ }^{1}$ Interestingly, a related type of automata on ordinals was used by Büchi [Büc65b, Büc65a] to study the decidability of the monadic theory of the ordinals.

${ }^{2}$ Generalized Büchi automata differ from Büchi automata [Büc62] in that they have a set of sets of accepting states rather than just one set of accepting states.
} 
- $\Delta \subseteq S \times \Sigma \times S$ is a transition relation,

- $s_{0} \in S$ is the starting state, and

- $\mathcal{F}=\left\{F_{1}, \ldots, F_{k}\right\} \subseteq 2^{S}$ is a set of sets of accepting states.

Generalized Büchi automata are used to define languages of $\omega$-words, i.e. functions from the ordinal $\omega$ to the alphabet $\Sigma$. Intuitively, a word is accepted by a Generalized Büchi automaton if the automaton has an infinite execution that intersects infinitely often each of the sets $F_{j} \in \mathcal{F}$.

Formally, we define the concept of a computation of $A$ over an $\omega$-word, i.e. a function from the ordinal $\omega$ to the alphabet $\Sigma$. A computation $\sigma$ of $A$ over an $\omega$-word $w=a_{1} a_{2} \ldots$ is an $\omega$-sequence $\sigma=s_{0}, s_{1}, \ldots$ (i.e. a function from $\omega$ to $S)$ where $\left(s_{i-1}, a_{i}, s_{i}\right) \in \Delta$, for all $i \geq 1$. A computation $\sigma=s_{0}, s_{1}, \ldots$ is accepting if, for each $F_{j} \in \mathcal{F}$, there is some state in $F_{j}$ that repeats infinitely often, i.e. for some $s \in F_{j}$ there are infinitely many $i \in \omega$ such that $s_{i}=s$. The $\omega$-word $w$ is accepted by $A$ if there is an accepting computation of $A$ over $w$. The set of $\omega$-words accepted by $A$ is denoted $L_{\omega}(A)$.

An automaton $A_{P}$ representing the joint behavior of the processes $P_{i}$ can be computed by taking the product of the automata describing each process, actions that appear in several processes are synchronized, others are interleaved. Formally, the product $(\times)$ of two (generalization to the product of $n$ automata is immediate) generalized Büchi automata $A_{1}=\left(\Sigma_{1}, S_{1}, \Delta_{1}, s_{01}, \mathcal{F}_{1}\right)$ and $A_{2}=$ $\left(\Sigma_{2}, S_{2}, \Delta_{2}, s_{02}, \mathcal{F}_{2}\right)$ is the automaton $A=\left(\Sigma, S, \Delta, s_{0}, \mathcal{F}\right)$ defined by

- $\Sigma=\Sigma_{1} \cup \Sigma_{2}$

- $S=S_{1} \times S_{2}, s_{0}=\left(s_{01}, s_{02}\right)$,

- $\mathcal{F}=\bigcup_{F_{j} \in \mathcal{F}_{1}}\left\{F_{j} \times S_{2}\right\} \cup \bigcup_{F_{j} \in \mathcal{F}_{2}}\left\{S_{1} \times F_{j}\right\}$

- $((s, t), a,(u, v)) \in \Delta$ when

- $a \in \Sigma_{1} \cap \Sigma_{2}$ and $(s, a, u) \in \Delta_{1}$ and $(t, a, v) \in \Delta_{2}$,

$-a \in \Sigma_{1} \backslash \Sigma_{2}$ and $(s, a, u) \in \Delta_{1}$ and $v=t$,

$-a \in \Sigma_{2} \backslash \Sigma_{1}$ and $u=s$ and $(t, a, v) \in \Delta_{2}$.

Note that with this definition, the product automaton can have an infinite accepting computation that corresponds to a finite computation of some (but not all) of its components. Indeed, if a component $i$ has a state $s$ such that 
$s \in F_{j}$ for all $F_{j} \in \mathcal{F}_{i}$, then an infinite computation of the product in which component $i$ stays indefinitely in state $s$ will appear as accepting. This is a counterintuitive consequence of the straightforward definition we have chosen for the product. To avoid this, we adopt the following restriction on the acceptance conditions of the generalized Büchi automata we will use.

- either the acceptance condition is vacuous $(\mathcal{F}=\emptyset)$, in which case the automaton can have either finite or infinite computations, or

- the set $\mathcal{F}$ contains at least two disjoint components, in which case the product automaton cannot have an accepting computation corresponding to a finite computation of the automaton.

For a given generalized Büchi automaton, it is quite straightforward to construct an equivalent automaton that satisfies this restriction. In programming terms, the restriction is a form of fairness condition imposed on the processes with nonvacuous acceptance conditions: their executions must be infinite (executions that might legitimately not be infinite can be modelled by using an additional "idling" action).

To obtain a model-checking procedure, the only fact we need about lineartime temporal logic is that, for each formula $f$, it is possible to build a generalized Büchi automaton $A_{f}$ that accepts exactly the infinite words satisfying the temporal formula $f$ (the alphabet of this automaton is $2^{P}$ where $P$ is the set of propositions appearing in the formula $f$ ) [WVS83, VW86, Wol89]. This construction is exponential in the length of the formula, but this is usually not a problem since the formulas to be checked are quite short and since the algorithm often behaves much better than its upper bound. The model-checking procedure is then the following:

1. Build the finite-automaton on infinite words for the negation of the formula $f$ (one uses the negation of the formula as this yields a more efficient algorithm). The resulting automaton is $A_{\neg f}$.

2. Compute the product $A_{G}=\prod_{1 \leq i \leq n} A_{i} \times A_{\neg f}$ (in practice only the reachable states of this product).

3. Check if the automaton $A_{G}$ is nonempty.

To check if the automaton $A_{G}$ is nonempty, it is sufficient to check that its graph contains a strongly connected component that is reachable from the 
initial state and that includes a state from each of the sets $F_{j}$ of its set $\mathcal{F}$ of accepting sets. This can be done with a linear-time algorithm [AHU74]. The complexity of this model-checking method is thus determined by the size of $A_{G}$. Note that model checking is often said to be of complexity "linear in the size of the program" which is correct if one measures the size of the program as the

size of $\prod_{1 \leq i \leq n} A_{i}$. In practice, the limits of all model-checking methods come from the often excessive size of this product. The frustrating fact is that a lot of this excessive size is unnecessary: it is due to the modelling of concurrency by interleaving. This is what we are tempting to eliminate. Let us therefore turn to partial-order semantics.

\section{Partial-Order Semantics and Trace Automata}

In partial-order semantics, the possible behaviors of a concurrent system are described in terms of partial orders instead of sequences. More precisely, we use Mazurkiewicz's traces [Maz86] as semantic model. We briefly recall some basic notions of Mazurkiewicz's trace theory.

Definition 3.1 $A$ concurrent alphabet is a pair $\Sigma=(A, D)$ where $A$ is a finite set of symbols, called the alphabet of $\Sigma$, and where $D$ is a binary, symmetrical, and reflexive relation on $A$ called the dependency in $\Sigma$.

$I_{\Sigma}=A^{2} \backslash D$ stands for the independency in $\Sigma$.

Definition 3.2 Let $\Sigma=(A, D)$ be a concurrent alphabet, let $A^{*}$ represent the set of all finite sequences (words) of symbols in A, let $\cdot$ stand for the concatenation operation, and let $\varepsilon$ denote the empty word. We define the relation $\equiv_{\Sigma}$ as the least congruence in the monoid $\left[A^{*} ; \cdot, \varepsilon\right]$ such that

$$
(a, b) \in I_{\Sigma} \Rightarrow a b \equiv_{\Sigma} b a .
$$

The relation $\equiv_{\Sigma}$ is referred to as the trace equivalence over $\Sigma$.

Definition 3.3 Equivalence classes of $\equiv_{\Sigma}$ are called traces over $\Sigma$.

The trace characterized by a word $w$ and a concurrent alphabet $\Sigma$ is denoted by $[w]_{\Sigma}$. Thus a trace over a concurrent alphabet $\Sigma=(A, D)$ represents a set of words defined over $A$ that only differ by the order of adjacent symbols which 
are independent according to $D$. For instance, if $a$ and $b$ are two symbols of $A$ which are independent according to $D$, the trace $[a b]_{\Sigma}$ represents the two words $a b$ and $b a$. A trace is an equivalence class of words.

Let us now return to a concurrent program described as the composition of $n$ finite-state transition systems $A_{i}$ and of a property $f$ represented by the automaton $A_{\neg f}$. From now on, $A_{\neg f}$ will be denoted by $A_{n+1}$. Let $\Delta \subseteq S \times \Sigma \times S$ denote the transition relation of the product $A_{G}$ of these automata.

For each transition $t=\left(\mathbf{s}, a, \mathbf{s}^{\prime}\right) \in \Delta$ with $\mathbf{s}=\left(s_{1}, s_{2}, \ldots, s_{n+1}\right)$ and $\mathbf{s}^{\prime}=$ $\left(s_{1}^{\prime}, s_{2}^{\prime}, \ldots, s_{n+1}^{\prime}\right)$, the sets (by extension, we consider the states of $A_{G}$ as sets in the following definitions ${ }^{3}$ )

$\mathbf{-}^{\bullet} t=\left\{s_{i} \in \mathbf{s}:\left(s_{i}, a, s_{i}^{\prime}\right) \in \Delta_{i}\right\}$

- $t^{\bullet}=\left\{s_{i}^{\prime} \in \mathbf{s}^{\prime}:\left(s_{i}, a, s^{\prime}{ }_{i}\right) \in \Delta_{i}\right\}$

$-t^{\bullet}=\bullet t \cup t^{\bullet}$

are called respectively the preset, the postset and the proximity of the transition $t$. Intuitively, the preset, resp. the postset, of a transition $t=\left(\mathbf{s}, a, \mathbf{s}^{\prime}\right)$ of $A_{G}$ represents the states of the $A_{i}$ 's that synchronize together on $a$, respectively before and after this transition. We say that the $A_{i}$ 's with a nonempty preset and postset for a transition $t$ are active for this transition.

Two transitions $t_{1}=\left(\mathbf{s}_{1}, a_{1}, \mathbf{s}_{1}^{\prime}\right), t_{2}=\left(\mathbf{s}_{2}, a_{2}, \mathbf{s}_{2}^{\prime}\right) \in \Delta$ are said to be equivalent (notation $\equiv$ ) iff

$$
{ }^{\bullet} t_{1}=\bullet t_{2} \wedge t_{1}^{\bullet}=t_{2}^{\bullet} \wedge a_{1}=a_{2}
$$

Intuitively, two equivalent transitions represent the same transition but correspond to distinct occurrences of this transition. These occurrences can only differ by the states of the $A_{i}$ 's that are not active for the transition. We denote by $T$ the set of equivalence classes defined over $\Delta$ by $\equiv$. By extension, we define the preset, resp. the postset, of an element of set $T$ as being the preset, resp. the postset, of all transitions in the corresponding equivalence class. From now on, "transition" will refer to an element of $T$ rather than of $\Delta$.

We define the dependency in $A_{G}$ as the relation $D_{A_{G}} \subseteq T \times T$ such that:

$$
\left(t_{1}, t_{2}\right) \in D_{A_{G}} \Leftrightarrow \bullet t_{1}^{\bullet} \cap \bullet t_{2}^{\bullet} \neq \emptyset .
$$

\footnotetext{
${ }^{3}$ We assume that the sets $S_{1}, \ldots, S_{n+1}$ (where $S_{i}$ is the set of states of $A_{i}$ ) are pairwise disjoint.
} 
The complement of $D_{A_{G}}$ is called the independency in $A_{G}$. If two independent transitions occur next to each other in a computation, the order of their occurrences is irrelevant, since they occur concurrently in this execution. (Note that there are other possible ways of defining the notion of dependency [GP93].)

Let $\Sigma_{A_{G}}=\left(T, D_{A_{G}}\right)$ be the concurrent alphabet associated with $A_{G}$ and let $L\left(A_{G}\right)$ be the language of finite words over $T$ accepted by $A_{G}$ (all states of $A_{G}$ considered accepting). In other words, $L\left(A_{G}\right)$ is the set of finite sequences of transitions that the system $A_{G}$ can perform from its initial state. We define the trace behavior of $A_{G}$ as the set of equivalence classes of $L\left(A_{G}\right)$ defined by the relation $\equiv_{\Sigma_{A_{G}}}$. These equivalence classes are called traces of $A_{G}$. Such a class (trace) corresponds to a partial order (i.e. a set of causality relations) and represents all its linearizations (words).

To describe the behavior of $A_{G}$ by means of traces rather than sequences, we need the dependency $D_{A_{G}}$ of $A_{G}$ and only one linearization for each trace of $A_{G}$. So, the behavior of $A_{G}$ is fully characterized by the dependency $D_{A_{G}}$ and an automaton which generates (at least) one linearization for each trace. We call such an automaton a trace automaton (denoted $A_{T}$ ) for $A_{G}$ [God90].

Formally, the language $L\left(A_{T}\right)$ accepted by a trace automaton $A_{T}$ satisfies the following relation:

$$
L\left(A_{G}\right)=\bigcup_{w \in L\left(A_{T}\right)} \operatorname{Pref}\left(\operatorname{lin}\left([w]_{\Sigma_{A_{G}}}\right)\right)
$$

where $\operatorname{lin}\left([w]_{\Sigma_{A_{G}}}\right)$ denotes the set of linearizations (words) of the trace (equivalence class) $[w]_{\Sigma_{A_{G}}}$ and $\operatorname{Pref}(w)$ denotes the prefixes of $w$.

In [God90] an algorithm for constructing a trace automaton corresponding to a concurrent program ${ }^{4}$ is given. To construct such an automaton $A_{T}$, we do not need to compute all the reachable states of $A_{G}$ : whenever several independent transitions are executable, we execute only one of these transitions in order to generate only one interleaving (linearization) of these transitions. By construction, $A_{T}$ is a "sub-automaton" of $A_{G}$ (i.e. the states of $A_{T}$ are states of $A_{G}$ and the transitions of $A_{T}$ are transitions of $A_{G}$ ). The order of the time complexity for the algorithm presented in [God90] is given by the number of transitions in $A_{T}$ times the maximum number of simultaneous executable

\footnotetext{
${ }^{4}$ In [God90] a concurrent program is represented by a contact-free one-safe P/T-net instead of a parallel composition of sequential processes as defined here; since the former is a more general formalism (it allows the modelling of process creation/deletion) than the latter, the algorithm described in [God90] is still applicable in the context considered here.
} 
transitions. In practice it turns out that building $A_{T}$ often requires much less time and memory than building $A_{G}$.

For instance, the behavior of a simple protocol like the 5-dining-philosophers problem (see [God90]) that would classically require the use of a state-graph $A_{G}$ containing 2163 states and 8770 transitions can be represented by a trace automaton $A_{T}$ containing only 72 states and 83 transitions.

\section{Using Trace Automata for Model Checking}

In order to use the results of Section 3 for doing model checking, we would like to be able to proceed as follows.

1. Build the finite-automaton on infinite words for the negation of the formula $f$. The resulting automaton is $A_{\neg f}$.

2. Compute the trace automaton $A_{T}$ corresponding to the concurrent executions of the processes $A_{i}, 1 \leq i \leq n$, and of the automaton $A_{\neg f}$.

3. Check if the automaton $A_{T}$ is nonempty.

Unfortunately, this is incorrect. First, there is an obvious reason that makes this incorrect which is that the trace automaton $A_{T}$ is not defined as an automaton on infinite words and hence does not have a set $\mathcal{F}$. However, this problem can be easily solved. Let $S_{G}$ and $S_{T}$ respectively be the set of states of $A_{G}$ and $A_{T}$. By construction, $S_{T} \subseteq S_{G}$. Let $\mathcal{F}_{G}=\left\{F_{1}, \ldots, F_{k}\right\}$ be the set of sets of accepting states of $A_{G}$. The set $\mathcal{F}_{T}$ of sets of accepting states of $A_{T}$ is then defined by $\mathcal{F}_{T}=\left\{F^{\prime}{ }_{1}, \ldots, F^{\prime}{ }_{k}\right\}$ with $F^{\prime}{ }_{i}=F_{i} \cap S_{T}$.

Even if we extend the definition of $A_{T}$ to include the set $\mathcal{F}_{T}$ defined above (let us call the result $A_{T}^{\infty}$ ), we still cannot use $A_{T}^{\infty}$ for model checking. Indeed it is quite possible that the automaton $A_{G}$ obtained by the traditional computation of the product accepts some infinite word whereas $A_{T}^{\infty}$ does not accept any infinite word. This might seem counter intuitive because one could expect that, if $A_{G}$ accepts some word $w$, then by permuting independent transitions of the computation accepting $w$, one would obtain an accepting computation of $A_{T}^{\infty}$ which would then be nonempty. This is actually true for finite computations but not for infinite computations. Indeed, consider two processes that are totally independent (their alphabets are completely disjoint). The trace automaton for these two processes can be one that allows any number of transitions of the first 
process followed by any number of transitions of the second process. This is is fine for finite computations, but for infinite computations, one will be left with either an infinite computation of the first process or one of the second process, but not an infinite computation of both processes. One can summarize this by saying that $A_{T}^{\infty}$ represents the infinite computations of all processes, but not the joint infinite computations of unsynchronized processes. The following example illustrates this situation. Consider the generalized Büchi automata $A$ and $A^{\prime}$ of Figures 1 and 2 where $\mathcal{F}=\left\{\left\{s_{1}\right\},\left\{s_{2}\right\}\right\}$ and $\mathcal{F}^{\prime}=\left\{\left\{s_{1}^{\prime}\right\},\left\{s_{2}^{\prime}\right\}\right\}$ respectively. A possible trace automaton $A_{T}^{\infty}$ is given in Figure 3. Its set of sets of accepting states is defined by $\mathcal{F}_{T}=\left\{\left\{\left(s_{1}, s_{0}^{\prime}\right),\left(s_{1}, s_{1}^{\prime}\right),\left(s_{1}, s_{2}^{\prime}\right)\right\},\left\{\left(s_{2}, s_{0}^{\prime}\right)\right\},\left\{\left(s_{1}, s_{1}^{\prime}\right)\right\}\right.$, $\left.\left\{\left(s_{1}, s_{2}^{\prime}\right)\right\}\right\}$. This automaton does not accept any word whereas there is a joint infinite execution of the automata $A$ and $A^{\prime}$ that would be accepted by the corresponding global automaton.

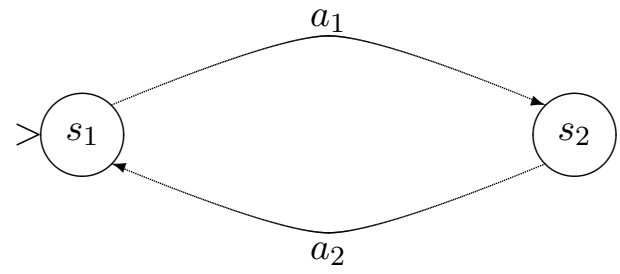

Figure 1: Generalized Büchi automaton $A$

We now formalize the above discussion. Let $A_{G}$ and $A_{T}^{\infty}$ be respectively the product automaton and the trace automaton obtained by composing the generalized Büchi automata $A_{i}, 1 \leq i \leq n+1$. Consider a computation of $A_{G}$ or $A_{T}^{\infty}$ on an infinite word $w$. One can view this computation as an infinite sequence of transitions, i.e., elements of set $T$ defined in Section 3. For any transition of $A_{G}$ or $A_{T}^{\infty}$, one can identify the automata $A_{i}$ that are active (as defined in Section 3) for this transition. This enables us to define the restriction of a computation of $A_{G}$ or $A_{T}^{\infty}$ to one of the components $A_{i}$.

Definition 4.1 Given a trace or product automaton A obtained by composing the generalized Büchi automata $A_{i}, 1 \leq i \leq n+1$, the restriction of a computation $\kappa$ of $A$ to the automaton $A_{i}$ (denoted $\kappa \mid A_{i}$ ) is the subsequence of $\kappa$ that contains only the transitions for which $A_{i}$ is active. 


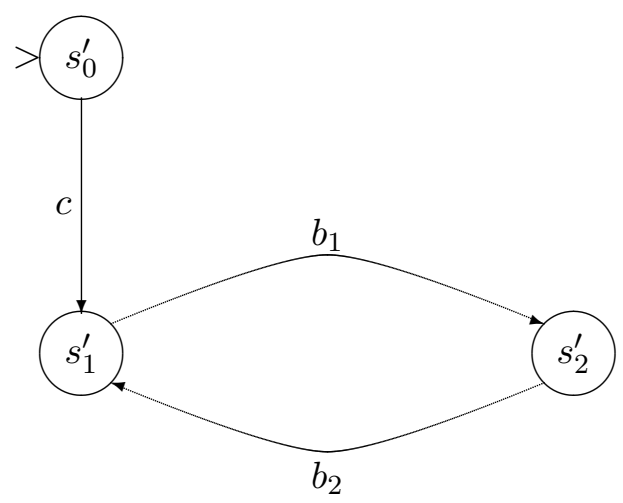

Figure 2: Generalized Büchi automaton $A^{\prime}$

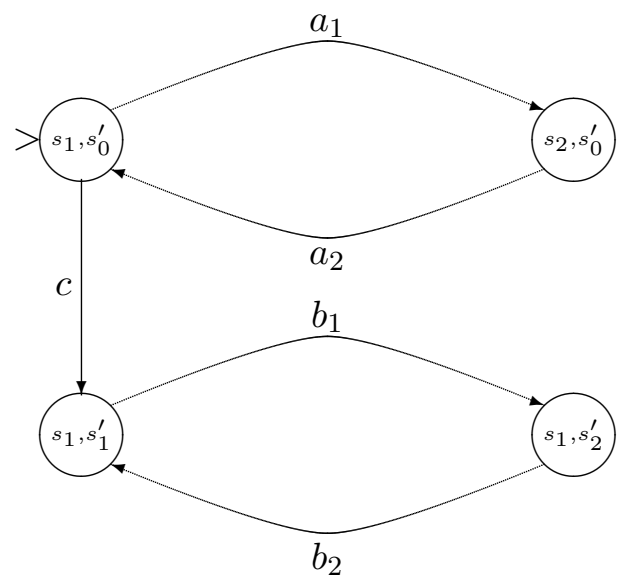

Figure 3: Trace automaton $A_{T}^{\infty}$ 
Note that the restriction of an infinite computation of $A_{G}$ or $A_{T}^{\infty}$ to an automaton $A_{i}$ can be finite. We have the following.

Theorem 4.1 Let $\kappa$ be a computation (finite or $\omega$-infinite) of the global automaton $A_{G}$ obtained by composing the automata $A_{i}, 1 \leq i \leq n+1$. Then, for every $A_{i}$, there is a computation $\kappa_{i}$ (finite or $\omega$-infinite) of the trace automaton $A_{T}^{\infty}$ such that $\kappa\left|A_{i}=\kappa_{i}\right| A_{i}$.

Proof: Consider a computation $\kappa$ of $A_{G}$. Consider then the computation $\kappa_{i}$ of $A_{T}^{\infty}$ which has the longest restriction $\kappa_{i} \mid A_{i}$ that is a prefix of $\kappa \mid A_{i}$ (if there are several such computations, choose one of these arbitrarily). Let $t_{i}$ be the first transition of $A_{i}$ in $\kappa$ that is not in this prefix. If there is no such transition, the theorem holds. Else, let us consider the prefix of $\kappa$ that ends with transition $t_{i}$. This finite computation is then the prefix of a trace of which, by definition of $A_{T}$, at least one linearization is generated by $A_{T}$. The projection on $A_{i}$ of any of these linearizations is $\kappa_{i} \mid A_{i} \cdot t_{i}$, which is longer than what we have assumed to be the longest projection on $A_{i}$ of a computation of $A_{T}^{\infty}$ that is a prefix of $\kappa \mid A_{i}$. Since computations of $A_{T}$ are trivially computations of $A_{T}^{\infty}$, we have a contradiction and the theorem follows.

Note that it is not true that there is a single computation $\kappa^{\prime}$ of $A_{T}^{\infty}$ such that $\kappa\left|A_{i}=\kappa^{\prime}\right| A_{i}$ for all $A_{i}$ 's. In spite of this, Theorem 4.1 lets us obtain an interesting result, namely that the trace automaton can be used for model checking in cases where only one of the components is required to have an infinite computation. This is the case if all but one of the automata $A_{i}$ have a vacuous accepting condition, i.e. have an empty set $\mathcal{F}$. This is proved in the following theorem.

Theorem 4.2 Let $A_{i}, 1 \leq i \leq n+1$ be generalized Büchi automata all but one of which have a vacuous accepting condition. Let $A_{G}$ and $A_{T}^{\infty}$ be the product and trace automata obtained by composing the automata $A_{i}$. Then, the automaton $A_{G}$ is nonempty (has at least one infinite accepting computation) iff the trace automaton $A_{T}^{\infty}$ is nonempty.

Proof: Assume $A_{G}$ has an infinite accepting computation $\kappa$ and let $A_{j}$ be the generalized Büchi automaton that has a nonvacuous acceptance condition. From Theorem 4.1, we know that there is a computation $\kappa_{j}$ of $A_{T}^{\infty}$ such that $\kappa\left|A_{j}=\kappa_{j}\right| A_{j}$. Since we have assumed in Section 2 that the product automaton 
$A_{G}$ cannot have an accepting computation corresponding to a finite computation of $A_{j}, \kappa \mid A_{j}$, and hence also $\kappa_{j} \mid A_{j}$, are infinite. Moreover, $\kappa \mid A_{j}$ intersects infinitely often each of the sets $F \in \mathcal{F}_{j}$ and, given that $\kappa_{j}\left|A_{j}=\kappa\right| A_{j}$, this is also the case for $\kappa_{j}$. This proves that $A_{T}^{\infty}$ is nonempty.

The other direction of the theorem is directly obtained from the immediate fact that all computations of the trace automaton are also computations of the global automaton.

In practice, Theorem 4.2 enables us to use the trace automaton for model checking in the cases where the program does not operate under some fairness hypothesis, or when the fairness hypothesis is incorporated into the formula to be verified. Indeed, in those circumstances, the automata representing the program will have vacuous accepting conditions and the automaton obtained from the formula to be checked will be the only one with a nonempty set $\mathcal{F}$.

\section{Automata on $(\omega \times n)$-words}

Trace automata do not adequately represent the $\omega$-computations of the components from which they are built because infinite computations cannot be concatenated. Actually, with the help of a little abstraction, infinite computations could very well be concatenated. One can simply think of computations whose length is an ordinal larger than $\omega$. Since we are only interested in the concatenation of a finite number of infinite computations we will only study computations of length $\omega \times n$ where $n \in \omega$. The definitions of Section 2 can be quite naturally extended to words and computations of length $\omega \times n$ (for other definitions of automata on ordinals, see [Büc65b, Büc65a]).

A word of length $\omega \times n$ over the alphabet $\Sigma$ is a function $w$ from the ordinal $\omega \times n$ to $\Sigma$. We use automata that are defined exactly as in Section 2 and simply change the definition of a computation. A computation of an automaton $A=\left(\Sigma, S, \Delta, s_{0}, \mathcal{F}\right)$ on a word $w$ of length $\omega \times n$ is a function $\sigma$ from $\omega \times n$ to $S$ that satisfies the following conditions:

1. $\sigma(0)=s_{0}$;

2. for each successor ordinal $\alpha+1 \in \omega \times n,(\sigma(\alpha), w(\alpha), \sigma(\alpha+1)) \in \Delta$;

3. for each limit ordinal $\lambda \in \omega \times n$, there is an infinite sequence of ordinals $\alpha$ whose limit is $\lambda$ such that $\sigma(\alpha)=\sigma(\lambda)$. 
The notions of accepting computation and accepted word are essentially unchanged. A computation $\sigma$ is accepting if, for each $F_{j} \in \mathcal{F}$, there is some state in $F_{j}$ that repeats infinitely often, i.e., for some $s \in F_{j}$ there are infinitely many $i \in \omega \times n$ such that $s_{i}=s$. The $\omega \times n$-word $w$ is accepted by $A$ if there is an accepting computation of $A$ over $w$. The set of $\omega \times n$ words accepted by $A$ is denoted $L_{\omega \times n}(A)$. Note that if an automaton accepts a word of length $\omega \times n$, $n \geq 1$, it also accepts a word of length $\omega \times n^{\prime}$ for all $n \leq n^{\prime}<\omega$.

Checking that $L_{\omega \times n}(A)$ is nonempty can be done by computing the maximal strongly connected components of $A$.

Theorem 5.1 Let $A=\left(\Sigma, S, \Delta, s_{0}, \mathcal{F}\right)$ be an automaton. Then, $L_{\omega \times n}(A) \neq \emptyset$ iff there is a sequence of nontrivial maximal strongly connected components $C_{1}, \ldots C_{n}$ in $A$ such that

- $C_{1}$ is accessible from $s_{0}$ and $C_{i+1}$ is accessible from $C_{i}$, for $1 \leq i<n$ and

- for each $F_{j} \in \mathcal{F}$, there is some $C_{i}$ such that $F_{j} \cap C_{i} \neq \emptyset$.

Proof: Assume that $A$ has an accepting computation $\kappa$ of length $\omega \times n$. Since $A$ is finite state, the first $\omega$-sequence of this computation must, from some point on, have all its states included in a nontrivial maximal strongly connected component $C_{1}$ of $A$. Similarly, the second $\omega$-sequence must start in a state of $C_{1}$ and must end in a component $C_{2}$ accessible from $C_{1}$ (it could actually be $C_{1}$ itself). Repeating the same line of thought for all $\omega$ sequences in $\kappa$ up to the $n$ th, one concludes the existence of the sequence of maximal strongly connected components $C_{i}, 1 \leq i \leq n$. Moreover, since $\kappa$ is accepting, $\kappa$ contains at least one state of each set $F_{j} \in \mathcal{F}$ infinitely often. And, since these states appear infinitely often, they must be in one of the components $C_{i}$. This proves that the condition given in the theorem is necessary.

To prove that it is sufficient, let us assume the existence of the sequence of connected components and construct an accepting computation. The first $\omega$-sequence in the computation starts in the initial state, has a finite prefix that leads it to the component $C_{1}$ and then goes infinitely often through all states of $C_{1}$. The second $\omega$-sequence starts from any state in $C_{1}$, has a finite prefix that leads to $C_{2}$ and then goes infinitely often through all states in $C_{2}$. The following $\omega$-sequences in the computation up to the $n$th are defined similarly. Since it goes through all states of all components $C_{i}$ infinitely often, this computation 
is clearly accepting.

The interesting aspect of the definitions we have just given is that if we consider the trace automaton as an automaton on words of length $\omega \times n$, then it represents all infinite computations of the combined automata. To prove this, we first establish a lemma.

Lemma 5.2 Let $\kappa$ be a finite computation of the global automaton $A_{G}$ obtained by composing the automata $A_{i}, 1 \leq i \leq n+1$. Then, there is a finite computation $\kappa^{\prime}$ of $A_{T}$ such that for all $1 \leq i \leq n+1, \kappa \mid A_{i}$ is a prefix of $\kappa^{\prime} \mid A_{i}$.

Proof: The lemma is a direct consequence of the definition of trace automata. Indeed, if $\kappa$ is a finite computation of $A_{G}$, then there is a representative of a trace that extends $\kappa$ that is a computation $\kappa^{\prime}$ of $A_{T}$. Thus, since adjacent transitions of a single process are never independent, we must have that $\kappa \mid A_{i}$ is a prefix of $\kappa^{\prime} \mid A_{i}$.

If we extend the notion of computation used in Section 4 to sequences of transitions of length $\omega \times n$, we have the following.

Theorem 5.3 Assume that the global automaton $A_{G}$ obtained by composing the automata $A_{i}, 1 \leq i \leq n+1$ has an accepting $\omega$-computation $\kappa$. Then, there is an accepting computation $\kappa^{\prime}$ of length at most $\omega \times(n+1)$ of the trace automaton $A_{T}^{\infty}$.

Proof: We use a pumping argument to prove this theorem. We start by considering a finite prefix $\kappa_{f}$ of $\kappa$ that is long enough to satisfy the following condition for each automaton $A_{i}$ that has a nonvacuous accepting condition. We describe the condition for a generic automaton $A_{i}$. Let $\mathcal{F}=\left\{F_{1}, \ldots, F_{k}\right\}$ be the accepting condition of $A_{i}$. For each set $F_{j}, 1 \leq j \leq k$, there is thus at least some state $s_{j} \in F_{j}$ that appears infinitely often in $\kappa$ (more precisely, this state appears in $\kappa$ as the component of the global state corresponding to $A_{i}$ ). This implies that by focusing on selected states of $\kappa$, one can identify infinitely often the sequence $s_{1}, s_{2}, \ldots, s_{k}$ (of $A_{i}$ components of the global state). The condition is that the sequence $s_{1}, s_{2}, \ldots, s_{k}$ can be selected from $\kappa_{f}$ at least as many times as there are states in $A_{T}$.

We then consider the computation $\kappa_{T f}$ of $A_{T}^{\infty}$ that satisfies the condition given in Lemma 5.2 for $\kappa_{f}$. This computation thus also satisfies the condition we have imposed on $\kappa_{f}$. Moreover, since the sequence $s_{1}, s_{2}, \ldots, s_{k}$ appears at 
least as many times as there are states in $A_{T}$, each of the states $s_{j}$ must appear at least twice as component of the same state of $A_{T}$. Thus $\kappa_{T f}$ contains, for instance, a subsequence that starts in a global state $\mathbf{s}$ whose $A_{i}$ component is $s_{1}$, that goes through at least one state of each of the accepting sets $F_{j}$ of $A_{i}$ and that ends again in the state $\mathbf{s}$. We call such a subsequence of $\kappa_{T f}$ an $A_{i}$-complete subsequence. Note moreover that our assumption that each set $\mathcal{F}$ contains at least two components implies that $A_{i}$-complete subsequences are nontrivial (contain at least one transition). We have thus established that $\kappa_{T f}$ contains at least one $A_{i}$-complete subsequence for each of the automata $A_{i}$ with nonvacuous accepting conditions.

The next step is to choose in $\kappa_{T f}$ an $A_{i}$-complete subsequence for each $A_{i}$ with a nonvacuous accepting computation and to sort these by order of appearance of their first state. Let $\sigma_{1}, \sigma_{2}, \ldots, \sigma_{\ell}, \ell \leq n+1$, be the $A_{i}$-complete subsequences taken in this order and let $\mathbf{s}_{1}, \mathbf{s}_{2}, \ldots \mathbf{s}_{\ell}$ be their respective first states (these states thus appear in $\kappa_{T f}$ in that order) and let $\mathbf{s}_{0}$ be the first state of $\kappa_{T f}$. Then, if we denote by $\left[\mathbf{s}_{i}, \mathbf{s}_{j}\right]$ the portion of $\kappa_{T f}$ that is included between $\mathbf{s}_{i}$ and $\mathbf{s}_{j}$ the following is an accepting computation of $A_{T}^{\infty}$ of length $\omega \times \ell\left(w^{\omega}\right.$ represents an infinite repetition of the word $\left.w\right)$

$$
\left[\mathbf{s}_{0}, \mathbf{s}_{1}\right] \sigma_{1}^{\omega}\left[\mathbf{s}_{1}, \mathbf{s}_{2}\right] \sigma_{2}^{\omega}\left[\mathbf{s}_{2}, \mathbf{s}_{3}\right] \sigma_{3}^{\omega} \cdots\left[\mathbf{s}_{\ell-1}, \mathbf{s}_{\ell}\right] \sigma_{\ell}^{\omega}
$$

To use the trace automaton for model checking, we also need the converse of Theorem 5.3. However, this does not hold in general since it requires that a computation of length $\omega \times(n+1)$ be merged into a computation of length $\omega$ which is not always possible since only independent transitions can be interchanged. More precisely, if $A_{T}^{\infty}$ is empty, Theorem 5.3 guaranties that $A_{G}$ is also empty and hence that the program satisfies the property. If $A_{T}^{\infty}$ is nonempty and has a computation of length $\omega, A_{G}$ is also nonempty and the program does not satisfy the property. The difficult case is when $A_{T}^{\infty}$ has an accepting computation of length greater than $\omega$. A simple approach to deal with this situation is to reconstruct part of $A_{G}$ in order to determine whether the computation of $A_{T}^{\infty}$ that has been found is an artifact or actually corresponds to a computation of $A_{G}$. It might seem that reconstructing part of $A_{G}$ looses the advantage of the partial order approach, but note that this need not be done in all cases, and that the construction is limited to the accepting computation of $A_{T}^{\infty}$ that has been found. Concretely, one can do the partial construction of $A_{G}$ from the projections on the various processes of the sequence of strongly connected 
components of $A_{T}^{\infty}$ that defines an accepting computation.

The partial construction of $A_{G}$ can be avoided in even more cases if one first checks whether the accepting computation of $A_{T}^{\infty}$ satisfies a "separability" condition. Consider a computation of length $\omega \times(n+1)$. For each $\omega$-sequence in this computation, i.e. part of the computation corresponding to an interval $[\omega \times j, \omega \times(j+1)[$, we define the repeating part of this $\omega$-sequence as its suffix that only contains states that appear infinitely often. The rest of the $\omega$-sequence is then its finite prefix. We call a computation separable if for all $0 \leq i<j \leq n$, all transitions in the repeating part of $[\omega \times i, \omega \times(i+1)[$ are independent of all transitions in the finite prefix of $[\omega \times j, \omega \times(j+1)[$. We can then show that the converse of Theorem 5.3 holds for separable computations.

Theorem 5.4 Let $A_{i}, 1 \leq i \leq n+1$ be generalized Büchi automata. Let $A_{G}$ and $A_{T}^{\infty}$ be the product and trace automata obtained by composing the automata $A_{i}$. Then, if the trace automaton $A_{T}^{\infty}$ has at least one separable accepting computation of length at most $\omega \times(n+1)$, the automaton $A_{G}$ is nonempty (has at least one accepting computation).

Proof: Notice that if $A_{T}^{\infty}$ has a separable accepting computation of length $\omega \times(n+1)$, it has an accepting computation of the form

$$
\sigma_{0} \sigma_{0 r}^{\omega} \sigma_{1} \sigma_{1 r}^{\omega} \cdots \sigma_{n} \sigma_{n r}^{\omega}
$$

where $\sigma_{i}$ and $\sigma_{i r}$ are finite computations and where all transitions in $\sigma_{i r}$ are independent with respect to all transitions in $\sigma_{j}$ for $0 \leq i<j \leq n$. As a consequence the following is an accepting $\omega$-computation of $A_{G}$

$$
\sigma_{0} \sigma_{1} \cdots \sigma_{n}\left(\sigma_{0 r} \sigma_{1 r} \cdots \sigma_{n r}\right)^{\omega}
$$

A sufficient condition for $A_{T}^{\infty}$ to have a separable condition is that is has a sequence of strongly connected components as in the condition of Theorem 5.1 and furthermore that for all $1 \leq i<j \leq(n+1)$, the transitions appearing in $C_{i}$ are independent from those appearing in the path from $C_{j-1}$ to $C_{j}$. For instance, the trace automaton in Figure 3 has a separable accepting computation $\left(a_{1} a_{2}\right)^{\omega} c\left(b_{1} b_{2}\right)^{\omega}$ of length $\omega \times 2$.

In summary, the procedure for checking whether $A_{T}^{\infty}$ has a computation corresponding to a computation of $A_{G}$ is the following. We first determine if $A_{T}^{\infty}$ has a sequence of strongly connected components that satisfy the condition 
of Theorem 5.1. If there is no such sequence, $A_{G}$ is empty. If there is such sequence, we check whether it satisfies the separability condition above. If it does, $A_{G}$ is nonempty. In the remaining cases, a partial search of $A_{G}$ is required to obtain a definite answer. Finally, note that another possible approach would be to guaranty the existence of a separable computation of $A_{T}^{\infty}$ whenever $A_{G}$ has a computation by using a different construction of $A_{T}$. Indeed, in all above, the only property of trace automata we have used is property (1) given in Section 3. Specific constructions of trace automata often have additional properties and can be tailored to satisfy specific requirements.

\section{Conclusions and Comparison with Other Work}

The closest work to the one presented here is certainly that of Valmari [Val90]. His paper also addresses the problem of adapting to model checking a method that avoids considering all interleavings of independent events while generating the state space of a concurrent program. It is likewise based on linear-time temporal logic, but uses a different strategy from the one we presented here. In our approach, the fact that the order of actions that appear in the formula cannot be ignored while constructing the trace automaton is handled by treating the property as any other component of the concurrent program. In [Val90], the problem is solved by a less discriminating approach. Precisely, the use of the "next" temporal operator is disallowed and all transitions that can affect the truth value of any state predicate appearing in the formula are considered as dependent. Prohibiting "next" is indeed important in this approach since in the presence of this operator all transition could potentially affect the truth value of the formula and hence would have to be considered as dependent and this would annihilate any benefit coming from the use of a partial-order approach. In our paper, we do handle the full temporal logic, and, actually, we can also handle extended temporal logics like that of [Wol83]. However, it should be noted that our interpretation of "next" is different from the one that causes problems in the method used by Valmari: we interpret "next" as meaning "next action monitored by the formula" rather than "next state of the program".

The treatment of fairness properties is also an important difference between Valmari's approach and ours. In Valmari's approach, the only way to represent fairness conditions would be to incorporate them in the formula (which hence has the form fair $\supset$ property) whereas we represent them as Büchi conditions on the processes. The interaction of fairness conditions and partial-order methods 
is problematic since a fairness condition often concerns all processes involved in the program and hence introduces many dependencies which can wipe out the benefit of the approach. Our solution is to represent fairness assumptions in a distributed way, by assigning progress conditions to individual processes whenever possible. The drawback of this strategy is that it does not yield naturally to the expression of some fairness constraints.

A final element of comparison is the algorithm for computing an automaton that only represents "some" interleavings of concurrent events that lies at the heart of both approaches. First, note that an important advantage of the use of $\omega \times n$ automata is that no modification of an algorithm suitable for finite computations is necessary. On the other hand, in [Val90] the algorithm has to be modified, which increases the size of the state space that is generated. Furthermore, the technical ideas behind the constructions used in both approaches differ. Valmari uses an algorithm based on "Stubborn Sets", we use the construction of the "Trace Automaton" given in [God90]. This difference also influences the effectiveness of the model-checking methods. However, this influence is not extremely clear cut and is orthogonal to that of the strategy being used. It is quite possible that for some problems the "Trace Automaton" algorithm is best whereas for others the "Stubborn Sets" one is preferable. It is worth noticing that parts of both algorithms can be combined in order to achieve better reductions [WG93].

How good really is our method? It is hard to give a precise answer since it might be no better than interleaving methods when there is very tight coupling between the processes and dramatically better when there is no coupling between the processes. In the latter case, we could claim as is done in $\left[\mathrm{BCM}^{+} 90\right]$ that we can check systems with astronomical numbers of (interleaving semantics) states. Of course this should be taken with a grain of salt since the fact that checking only part of this enormous state space is sufficient indicates that most of the interleaving-semantics states are uninteresting. In $\left[\mathrm{BCM}^{+} 90\right]$ a similar phenomenon occurs, the difference being that the verification of large systems is made possible not by ignoring an irrelevant part of their state space, but by computing with an efficient symbolic representation of sets of states and transition relations.

The construction of trace automata of [God90] has been implemented and shows promising results which bodes well for the method described in this paper. Other work on the implementation and use of trace-automata-like techniques 
has also appeared. In [GW91b], model checking restricted to safety properties is considered. Several alternative "partial-order" verification algorithms are presented in [HGP92] and their performance on real-protocols is evaluated. Further results on the practicality of using trace-automata-like constructions are presented in [GHP92].

Finally, note that our method has the advantages of "on the fly verification" [CVWY90, JJ89, BFH90, HPOG89]. By this we mean that we build the automaton for the combination of the program and property without ever building the automaton for the program. Maybe surprisingly, this automaton is often smaller than the automaton for the program alone because the property acts as a constraint on the behavior of the program. Our method thus has a head start over methods that require the state graph of the program to be built.

\section{Acknowledgements}

We wish to thank Jerry Burch, David Dill, Wolfgang Thomas, Frits Vaandrager, Antti Valmari and anonymous referees for helpful comments on this paper. A preliminary version of this paper appeared in the Proceedings of the 6th Symposium on Logic in Computer Science [GW91a].

\section{References}

[ACD90] R. Alur, C. Courcoubetis, and D. Dill. Model-checking for real-time systems. In Proceedings of the 5th Symposium on Logic in Computer Science, pages 414-425, Philadelphia, June 1990.

[ACW90] S. Aggarwal, C. Courcoubetis, and P. Wolper. Adding liveness properties to coupled finite-state machines. ACM Transactions on Programming Languages and Systems, 12(2):303-339, 1990.

[AH90] R. Alur and T. Henzinger. Real-time logics: complexity and expressiveness. In Proceedings of the 5th Symposium on Logic in Computer Science, pages 390-401, Philadelphia, June 1990.

[AHU74] Alfred V. Aho, John E. Hopcroft, and Jeffrey D. Ullman. The Design and Analysis of Computer Algorithms. Addison Wesley, Reading, 1974.

[BCD85] M. Browne, E.M. Clarke, and D.L. Dill. Automatic circuit verification using temporal logic: Two new examples. In IEEE Int. Conf. on Computer Design: VLSI and Computers, Port Chester, October 1985. 
$\left[\mathrm{BCM}^{+} 90\right]$ J.R. Burch, E.M. Clarke, K.L. McMillan, D.L. Dill, and L.J. Hwang. Symbolic model checking: $10^{20}$ states and beyond. In Proceedings of the 5th Symposium on Logic in Computer Science, pages 428-439, Philadelphia, June 1990.

[BFH90] A. Bouajjani, J. C. Fernandez, and N. Halbwachs. On the verification of safety properties. Technical Report SPECTRE L12, IMAG, Grenoble, March 1990.

[Bro86] Michael C. Browne. An improved algorithm for the automatic verification of finite state systems using temporal logic. In Proceedings of the First Symposium on Logic in Computer Science, pages 260-266, Cambridge, June 1986.

[Büc62] J.R. Büchi. On a decision method in restricted second order arithmetic. In Proc. Internat. Congr. Logic, Method and Philos. Sci. 1960, pages 1-12, Stanford, 1962. Stanford University Press.

[Büc65a] J.R. Büchi. Decision methods in the theory of ordinals. Bull of the AMS, 71:767-770, 1965.

[Büc65b] J.R. Büchi. Transfinite automata recursions and weak second order theory of ordinals. In Proc. Internat. Congr. Logic, Method and Philos. Sci. 1964, pages 2-23, Amsterdam, 1965. North Holland.

[CES86] E.M. Clarke, E.A. Emerson, and A.P. Sistla. Automatic verification of finite-state concurrent systems using temporal logic specifications. ACM Transactions on Programming Languages and Systems, 8(2):244-263, January 1986.

[CG87] E. M. Clarke and O. Grümberg. Avoiding the state explosion problem in temporal logic model-checking algorithms. In Proc. 6th ACM Symposium on Principles of Distributed Computing, pages 294-303, Vancouver, British Columbia, August 1987.

[CGB86] E. M. Clarke, O. Grümberg, and M. C. Browne. Reasoning about networks with many identical finite-state processes. In Proc. 5th ACM Symposium on Principles of Distributed Computing, pages 240-248, Calgary, Alberta, August 1986.

[Cle90] R. Cleaveland. Tableau-based model checking in the propositional mucalculus. Acta Informatica, 27:725-747, 1990.

[CMB90] O. Coudert, J. C. Madre, and C. Berthet. Verifying temporal properties of sequential machines without building their state diagram. In Proc. 2nd Workshop on Computer Aided Verification, volume 531 of Lecture Notes in Computer Science, pages 23-32, Rutgers, June 1990. Springer-Verlag. 
[CVWY90] C. Courcoubetis, M. Vardi, P. Wolper, and M. Yannakakis. Memory efficient algorithms for the verification of temporal properties. In Proc. 2nd Workshop on Computer Aided Verification, volume 531 of Lecture Notes in Computer Science, pages 233-242, Rutgers, June 1990.

[CY90] C. Courcoubetis and M. Yannakakis. Markov decision processes and regular events. In Proc. 17th Int. Coll. on Automata Languages and Programming, volume 443, pages 336-349, Coventry, July 1990. Lecture Notes in Computer Science, Springer-Verlag.

[EL85a] E.A. Emerson and C.-L. Lei. Modalities for model checking: Branching time logic strikes back. In Proceedings of the Twelfth ACM Symposium on Principles of Programming Languages, pages 84-96, New Orleans, January 1985.

[EL85b] E.A. Emerson and C.-L. Lei. Temporal model checking under generalized fairness constraints. In Proc. 18th Hawaii International Conference on System Sciences, Hawaii, 1985.

[EL86] E.A. Emerson and C.-L. Lei. Modalities for model checking: Branching time logic strikes back. In Proceedings of the First Symposium on Logic in Computer Science, pages 267-278, Cambridge, June 1986.

[GHP92] P. Godefroid, G. J. Holzmann, and D. Pirottin. State space caching revisited. In Proc. 4th Workshop on Computer Aided Verification, volume 663 of Lecture Notes in Computer Science, pages 178-191, Montreal, June 1992. Springer-Verlag.

[God90] P. Godefroid. Using partial orders to improve automatic verification methods. In Proc. 2nd Workshop on Computer Aided Verification, volume 531 of Lecture Notes in Computer Science, pages 176-185, Rutgers, June 1990.

[GP93] P. Godefroid and D. Pirottin. Refining dependencies improves partialorder verification methods. In Proc. 5th Conference on Computer Aided Verification, volume 697 of Lecture Notes in Computer Science, pages 438449, Elounda, June 1993. Springer-Verlag.

[GS87] S. German and A.P. Sistla. Reasoning with many processes. In Proc. of the First Symposium on Logic in Computer Science, pages 138-152, Ithaca, June 1987.

[GS90] S. Graf and B. Steffen. Using interface specifications for compositional reduction. In Proc. 2nd Workshop on Computer Aided Verification, volume 531 of Lecture Notes in Computer Science, pages 186-196, Rutgers, June 1990. Springer-Verlag.

[GW91a] P. Godefroid and P. Wolper. A partial approach to model checking. In Proceedings of the 6th IEEE Symposium on Logic in Computer Science, pages 406-415, Amsterdam, July 1991. 
[GW91b] P. Godefroid and P. Wolper. Using partial orders for the efficient verification of deadlock freedom and safety properties. In Proc. 3rd Workshop on Computer Aided Verification, volume 575 of Lecture Notes in Computer Science, pages 332-342, Aalborg, July 1991.

[HGP92] G. J. Holzmann, P. Godefroid, and D. Pirottin. Coverage preserving reduction strategies for reachability analysis. In Proc. 12th IFIP WG 6.1 International Symposium on Protocol Specification, Testing, and Verification, pages 349-363, Lake Buena Vista, Florida, June 1992. North-Holland.

[HLP90] E. Harel, O. Lichtenstein, and A. Pnueli. Explicit-clock temporal logic. In Proceedings of the 5th Symposium on Logic in Computer Science, pages 402-413, Philadelphia, June 1990.

[HPOG89] N. Halbwachs, D. Pilaud, F. Ouabdesselam, and A.C. Glory. Specifying, programming and verifying real-time systems, using a synchronous declarative language. In Workshop on automatic verification methods for finite state systems, volume 407 of Lecture Notes in Computer Science, pages 213-231, Grenoble, June 1989.

[JJ89] C. Jard and T. Jeron. On-line model-checking for finite linear temporal logic specifications. In Workshop on automatic verification methods for finite state systems, volume 407 of Lecture Notes in Computer Science, pages 189-196, Grenoble, June 1989.

[KM89] R. P. Kurshan and K. McMillan. A structural induction theorem for processes. In Proceedings of the Eigth ACM Symposium on Principles of Distributed Computing, Edmonton, Alberta, August 1989.

[KP86] Y. Kornatzky and S. S. Pinter. A model checker for partial order temporal logic. EE PUB 597, Department of Electrical Enginering, Technion-Israel Institute of Technology, 1986.

[LP85] O. Lichtenstein and A. Pnueli. Checking that finite state concurrent programs satisfy their linear specification. In Proceedings of the Twelfth ACM Symposium on Principles of Programming Languages, pages 97-107, New Orleans, January 1985.

[Maz86] A. Mazurkiewicz. Trace theory. In Petri Nets: Applications and Relationships to Other Models of Concurrency, Advances in Petri Nets 1986, Part II; Proceedings of an Advanced Course, volume 255 of Lecture Notes in Computer Science, pages 279-324, 1986.

[Pen90] W. Penczek. Proving partial order properties using cctl. Proc. Concurrency and Compositionality Workshop, San Miniato, Italy, 1990.

[PL90] D. K. Probst and H. F. Li. Using partial-order semantics to avoid the state explosion problem in asynchronous systems. In Proc. 2nd Workshop 
on Computer Aided Verification, volume 531 of Lecture Notes in Computer Science, pages 146-155, Rutgers, June 1990.

[PW84] S. S. Pinter and P. Wolper. A temporal logic for reasoning about partially ordered computations. In Proc. 3rd ACM Symposium on Principles of Distributed Computing, pages 28-37, Vancouver, 1984.

[PZ86] A. Pnueli and L. Zuck. Probabilistic verification by tableaux. In Proceedings of the First Symposium on Logic in Computer Science, pages 322-331, Cambridge, June 1986.

[QS81] J.P. Quielle and J. Sifakis. Specification and verification of concurrent systems in cesar. In Proc. 5th Int'l Symp. on Programming, volume 137 of Lecture Notes in Computer Science, pages 337-351, 1981.

[RRSV87] J.L. Richier, C. Rodriguez, J. Sifakis, and J. Voiron. Verification in xesar of the sliding window protocol. In Proc. IFIP WG 6.1 7th Int. Conf. on Protocol Specification, Testing and Verification, pages 235-250, Zurich, 1987. North Holland.

[SW89] C. Stirling and D. Walker. Local model checking in the modal mu-calculus. In Proc. 15th Col. on Trees in Algebra and Programming. Lecture Notes in Computer Science, Springer-Verlag, 1989.

[Tha89] André Thayse and et al. From Modal Logic to Deductive Databases: Introducing a Logic Based Approach to Artificial Intelligence. Wiley, 1989.

[Val90] A. Valmari. A stubborn attack on state explosion. In Proc. 2nd Workshop on Computer Aided Verification, volume 531 of Lecture Notes in Computer Science, pages 156-165, Rutgers, June 1990.

[Val91] A. Valmari. Stubborn sets for reduced state space generation. In Advances in Petri Nets 1990, volume 483 of Lecture Notes in Computer Science, pages 491-515, Bonn, June 1991. Springer-Verlag.

[Var85] M. Vardi. Automatic verification of probabilistic concurrent finite-state programs. In Proc. 26th IEEE Symp. on Foundations of Computer Science, pages 327-338, Portland, October 1985.

[Var88] M. Vardi. A temporal fixpoint calculus. In Proc. 15th ACM Symp. on Principles of Programming Languages, pages 250-259, San Diego, January 1988.

[Var89] M. Vardi. Unified verification theory. In B. Banieqbal, H. Barringer, and A. Pnueli, editors, Proc. Temporal Logic in Specification, volume 398, pages 202-212. Lecture Notes in Computer Science, Springer-Verlag, 1989.

[VW86] M.Y. Vardi and P. Wolper. An automata-theoretic approach to automatic program verification. In Proceedings of the First Symposium on Logic in Computer Science, pages 322-331, Cambridge, June 1986. 
[WG93] P. Wolper and P. Godefroid. Partial-order methods for temporal verification. In Proc. CONCUR '93, volume 715 of Lecture Notes in Computer Science, pages 233-246, Hildesheim, August 1993. Springer-Verlag.

[WL89] P. Wolper and V. Lovinfosse. Verifying properties of large sets of processes with network invariants. In Automatic Verification Methods for Finite State Systems, Proc. Int. Workshop, Grenoble, volume 407, pages 68-80, Grenoble, June 1989. Lecture Notes in Computer Science, Springer-Verlag.

[Wol83] P. Wolper. Temporal logic can be more expressive. Information and Control, 56(1-2):72-99, 1983.

[Wol89] P. Wolper. On the relation of programs and computations to models of temporal logic. In B. Banieqbal, H. Barringer, and A. Pnueli, editors, Proc. Temporal Logic in Specification, volume 398 of Lecture Notes in Computer Science, pages 75-123, 1989.

[WVS83] P. Wolper, M.Y. Vardi, and A.P. Sistla. Reasoning about infinite computation paths. In Proc. 24th IEEE Symposium on Foundations of Computer Science, pages 185-194, Tucson, 1983. 